\title{
Novel Noncrossing Y-Stent Technique Using Tapered Proximal End of a Solitaire AB Stent for Coil Embolization of Wide-Neck Bifurcation Aneurysms
}

\author{
Hyon-Jo Kwon, Jeong-Wook Lim, Hyoung Soo Byoun, Hyeon-Song Koh \\ Department of Neurosurgery, Daejeon-Chungnam Regional Cerebrovascular Center, Chungnam National University Hospital, Chungnam \\ National University Medical School, Daejeon, Korea
}

The crossing Y-stent method is one of the indispensable techniques to achieve sufficient neck coverage during coil embolization of bifurcation aneurysms with a wide neck and/or branch incorporation. However, the inevitable hourglass-like expansion of the second stent at the crossing point can result in insufficient vessel wall apposition, reduced aneurysm neck coverage, delayed endothelialization, and subsequent higher risks of acute or delayed thrombosis. It also interferes with engagement of the microcatheter into the aneurysm after stent installation. We expected to be able to reduce these disadvantages by installing a noncrossing type $Y$-stent using the Solitaire $A B$ stent, which is fully retrievable with a tapered proximal end. Here we report the techniques and two successful cases.

Key Words : Intracranial aneurysm · Treatment · Stents.

\section{INTRODUCTION}

The crossing Y-stent technique is a stenting method based on the installation of an additional stent towards a second branch through the struts of the first stent at the bifurcating branching segment, resulting in a Y-shaped stent ${ }^{10)}$. This technique enables us to coil embolize bifurcation-type aneurysms with a wide neck and/or branch incorporation. Although there are technical difficulties compared to the simple conventional stenting technique, previous reports analyzing this technique reported an acceptable complication rate, and it has contributed to favorable long-term aneurysmal occlusion rates $^{2,4,12)}$. However, the Y-stent structure has several disadvantages due to the inevitable crossing point in the vessel lumen. In addition to the difficulty in passing the microwire and microcatheter through the first stent strut for the second stent delivery, crossing of two stents necessarily causes incomplete expansion and hourglass-like stenosis of the second stent, resulting in insufficient vessel wall apposition and reduced aneurysmal neck coverage. The crowded struts in the center of the vessel lumen create a mechanical obstacle to microcatheter dome selection for coil packing. Most of all, we cannot expect endothelialization of this portion of the stent, which can be a life-long cause of acute or chronic thrombosis ${ }^{2,4,6,7,12)}$. Although

- Received : December 11, 2019 •Revised : February 19, 2020 •Accepted : March 30, 2020

- Address for reprints : Hyeon-Song Koh

Department of Neurosurgery, Chungnam National University Hospital, Chungnam National University Medical School, 282 Munhwa-ro, Jung-gu, Daejeon 35015, Korea Tel : +82-42-280-7369, Fax : +82-42-280-7363, E-mail : kohhs@cnu.ac.kr, ORCID : https://orcid.org/0000-0003-2659-5535

This is an Open Access article distributed under the terms of the Creative Commons Attribution Non-Commercial License (http://creativecommons.org/licenses/by-nc/4.0) which permits unrestricted non-commercial use, distribution, and reproduction in any medium, provided the original work is properly cited. 
several modified techniques to overcome these disadvantages have been published ${ }^{1,3,8}$, there are still remaining concerns and technical difficulties, especially for beginning providers.

We used the Solitaire AB stent (Medtronic, Irvine, CA, USA), which has been less commonly used in the Y-stenting technique, to reduce these shortcomings. The Solitaire stent, unlike other tubular shaped and closed-cell stents, has a rolling sheet-like structure and tapered proximal portion between the working zone of the stent and the proximal marker ${ }^{5}$. This proximal tapered portion, measuring approximately $1 \mathrm{~cm}$ regardless of working zone length, has much less surface area than the working zone of the stent and thus is relatively more flexible. The authors took advantages of these unique features of the Solitaire stent for noncrossing and reduced overlap Ystenting, and successfully treated two bifurcation-type aneurysms with very wide necks, as reported in this article.

\section{ENDOVASCULAR TECHNIQUES}

1) The same preparation was performed for typical stent-assisted coil embolization under general anesthesia and full heparinization. 2) The working angles and vessel segments were determined for the deployment of the two stents using a rotational angiogram and 3D reconstruction software. 3) The Solitaire stent was deployed from one of the bifurcations as the proximal end of the stent working zone aligned in front of the aneurysmal neck (Fig. 1A). 4) The status of the Solitaire stent deployment was confirmed, particularly aneurysmal neck coverage and the direction of the tapered proximal end, using flat-panel detector computed tomography (vasoCT, Philips Healthcare, Best, the Netherlands) or rotational angiogram. 5) The second stent was deployed from the other branch to the proximal vessel through the aneurysm neck after confirming that the working zone of the previous Solitaire stent covered the aneurysmal neck suitably. It was also confirmed
(A)

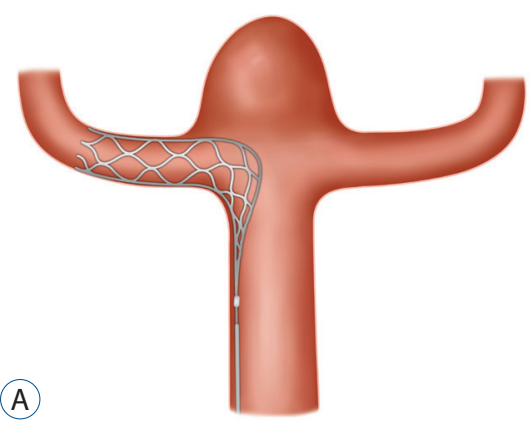

(D)

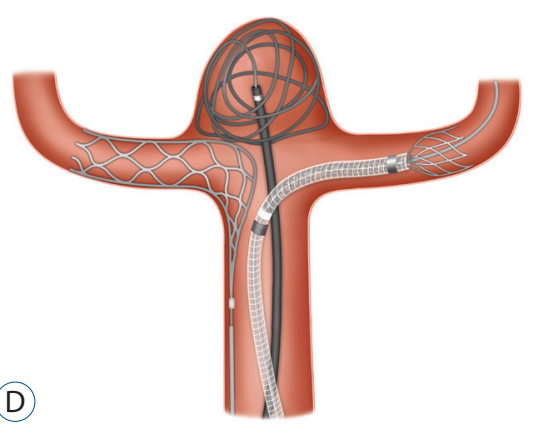

(B)
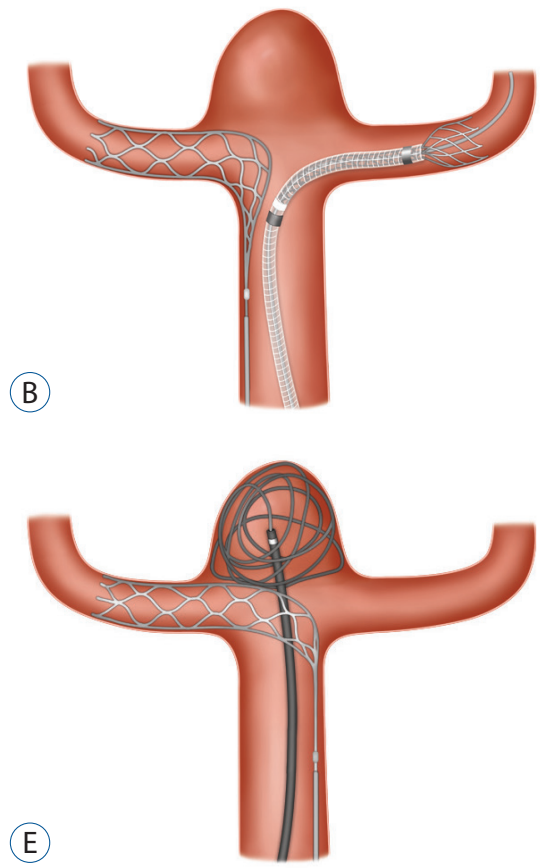

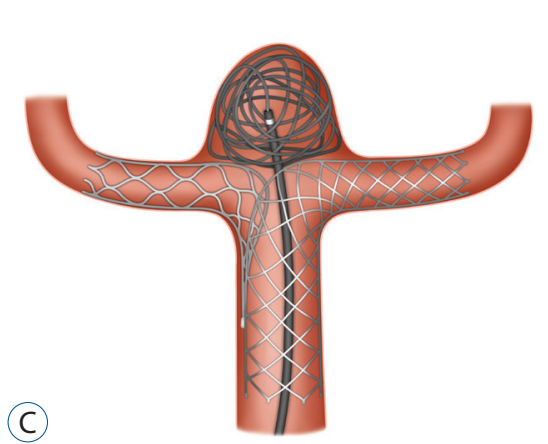

(C)

Fig. 1. Schematic diagram of the noncrossing Y-stent technique with the Solitaire AB stent. $A$ : The Solitaire $A B$ stent is deployed in one of the bifurcation branches. Note that the proximal part of the stent working zone is aligned with the aneurysm neck. $B$ : The second stent is to be deployed in the other branch. No obstacle was found on the passage of the second microcatheter and stent. C : After full deployment of the second stent that accomplishes a noncrossing $\mathrm{Y}$ design, sufficient aneurysmal neck coverage for coil filling is achieved. A microcatheter for coil filling is inserted inside the aneurysmal dome after detachment of the Solitaire stent, and a frame coil was deployed. D : Variation of this technique. A microcatheter for coil filling can be jailed according to the operator's discretion when the parent artery lumen is sufficient. E : A case in which the proximal tapered end of the Solitaire AB stent is directed at the origin of the opposite branch. By gently pushing of the delivery shaft, the proximal tapered end portion can be installed like the upper portion of the T-PulseRider, and better neck coverage can be achieved. Coil filling with single or multiple catheters may be attempted in this situation without second stent deployment. 

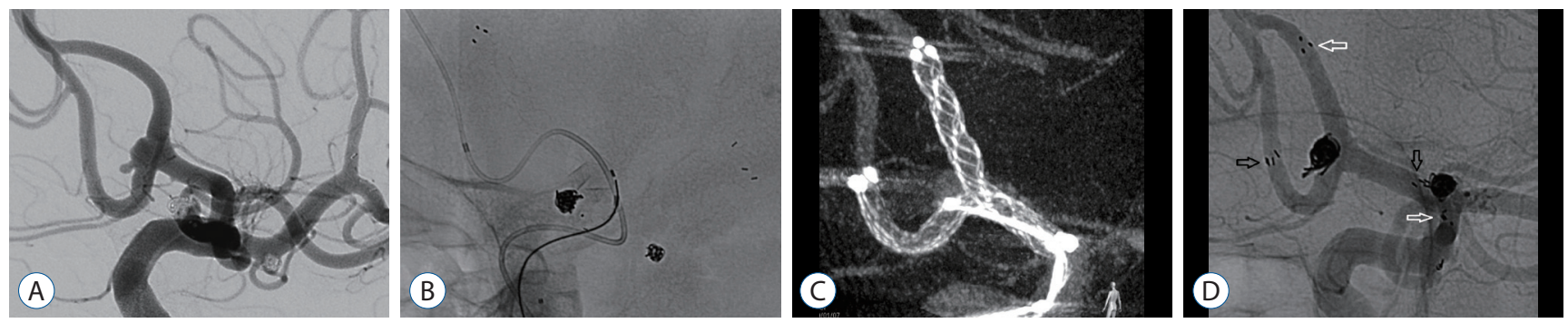

Fig. 2. This patient has three aneurysms; two in the middle cerebral artery have been occluded with conventional stent-assisted coil embolizations. A : Initial angiogram shows a shallow aneurysm with bleb at the bifurcating type anterior communicating artery. B : A Solitaire AB ( $4 \times 20 \mathrm{~mm})$ stent is deployed first and a microcatheter for the second stent (Enterprise $2 \times 30 \mathrm{~mm}$ ) is delivered to the contralateral distal anterior cerebral artery. $C$ : Reconstructed flat panel detector computed tomography image after successful deployment of the two stents and microcatheter selection in the aneurysm dome showing adequate neck coverage without crossing of stents. D : Final native angiogram after complete coil packing in the aneurysm including the bleb. Proximal and distal markers of Solitaire AB (white arrows) and Enterprise2 (black arrows) stents are indicated.

that the proximal tapered end did not enter the opposite branch origin and would not hinder the passage of the second microcatheter for the second stent (Fig. 1B). 6) The final status of the Y-stent installation was re-confirmed using vasoCT or rotational angiogram, based on the operator's discretion. And 7) the microcatheter for coil packing could be catheterized (Fig. 1C, case 1) after the detachment of the Solitaire stent or jailed (Fig. 1D, case 2) according to the parent vessel lumen capacity and operator's discretion. The coil filling could be performed under new working angles after completion of stent deployment.

\section{Case 1}

A woman was admitted to our hospital for coil embolization of multiple unruptured small aneurysms. Under general anesthesia and heparinization, two small middle cerebral artery aneurysms were embolized first using stent assistance. For the bifurcating type anterior communicating artery aneurysm with a depth-to-neck ratio of 0.41, the Prowler Select plus microcatheter (Cordis Corporation, Bridgewater, NJ, USA) was delivered to the ipsilateral distal anterior cerebral artery (ACA). After delivery through the microcatheter, the Solitaire AB stent $(4 \times 20 \mathrm{~mm})$ was deployed as planned previously. Using rotational angiogram, we confirmed that the proximal end of the stent working zone was aligned with the neck of the aneurysm and the proximal tapered end was not hindering the passage of the microcatheter for the second stent. During the delivery of the second Prowler Select plus microcatheter to the other ACA branch, no significant interruption was noticed and an Enterprise 2 stent $(4 \times 30 \mathrm{~mm}$, Codman Neurovascular, Raynham, MA, USA) was deployed successfully from contralateral A2 to A1 in front of the aneurysm neck. After selection of the aneurysm dome with an Excelsior10 microcatheter (Stryker, Fremont, CA, USA), framing and filling coils were packed until the aneurysm was completely obliterated (Fig. 2). No acute or delayed ischemic or other complications were identified during 8 months' outpatient clinic follow-up with dual anti-platelet medication, and no evidence of remnant aneurysm was found on magnetic resonance angiography 6 months after the procedure.

\section{Case 2}

A woman was admitted to our department for coil embolization of an incidental distal ACA aneurysm arising at the origin of trifurcating branches. The depth was $3.7 \mathrm{~mm}$ and the neck was $7.0 \mathrm{~mm}$. The procedure was carried out according to the usual protocol under general anesthesia. The Prowler Select plus microcatheter was delivered to the distal segment of one of the three branches and a Solitaire AB stent $(4 \times 15 \mathrm{~mm})$ was delivered in it. After the Solitaire stent was deployed as planned, we performed vasoCT to confirm the positioning of the stent, particularly at the aneurysm neck and the proximal tapered segment. During passage of the Excelsiorl0 microcatheter for the second stent, no obstacle was identified around the aneurysm neck segment of the distal ACA. After deployment of a Neuroform Atlas stent $(4.5 \times 30 \mathrm{~mm}$; Stryker, Kalamazoo, MI, USA) from the distal to proximal part of the parent vessel via the aneurysm neck, we again performed vasoCT to confirm the deployment status of the stents. Coil packing through the previously jailed Excelsior10 microcatheter was carried out uneventfully and successfully, and final angiogram showed a neck remnant only (Fig. 3). The patient showed no evidence of com- 

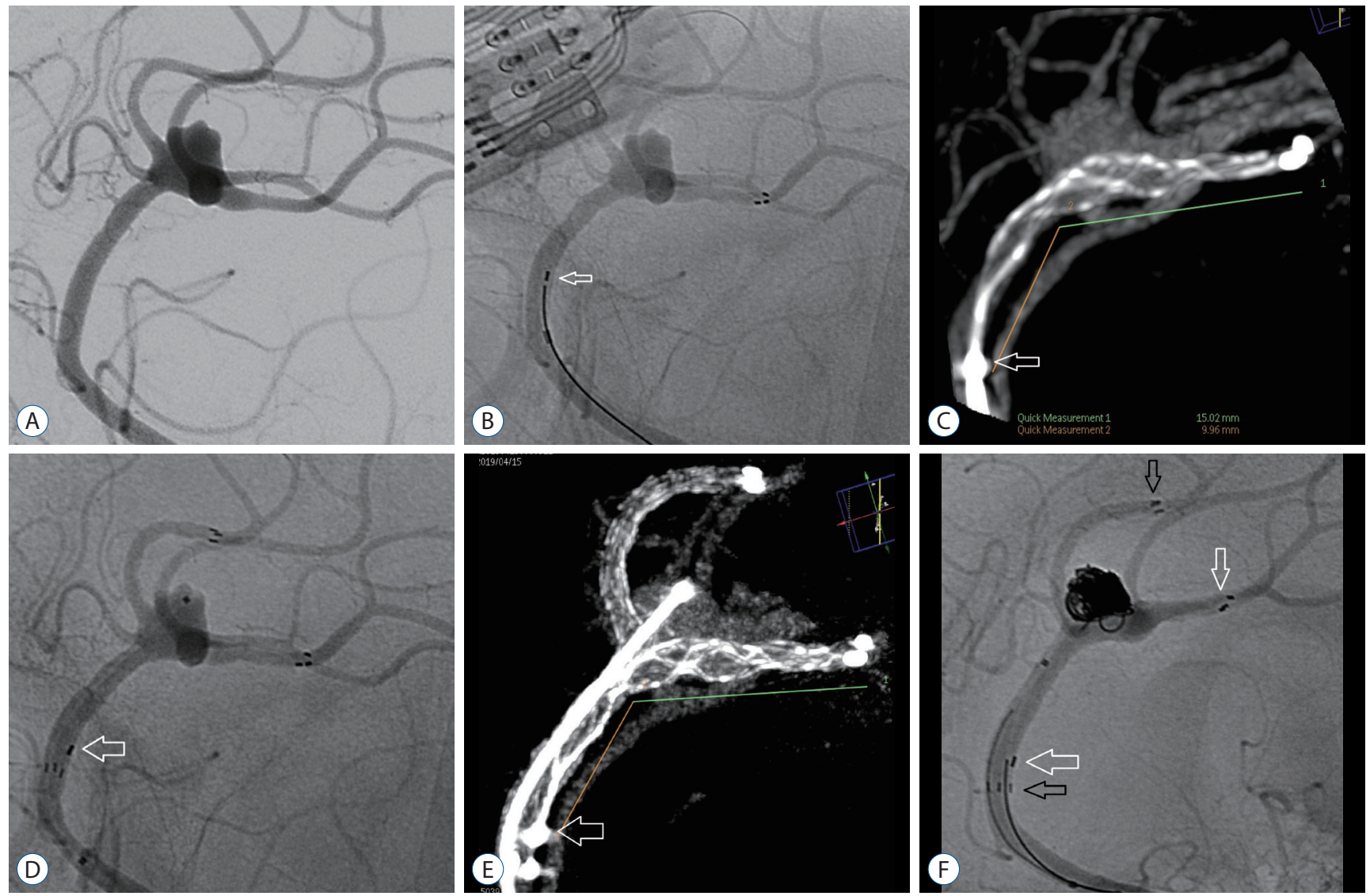

Fig. 3. A patient with wide-neck aneurysm at the distal anterior cerebral artery trifurcation site. A : Initial angiogram. B : The Solitaire AB (4x $15 \mathrm{~mm})$ stent is deployed in one of the trifurcation branches as planned. The proximal marker of the stent is noted (arrow). C : Reconstructed flat panel detector computed tomography (CT) image after deployment of the Solitaire $A B(4 \times 15 \mathrm{~mm})$ stent, showing that the proximal end of the stent working zone is aligned at the aneurysm neck. Measurement shows an approximately $15 \mathrm{~mm}$ - and $10 \mathrm{~mm}$-long working zone (green line) and proximal tapered end portion (orange line) of the Solitaire stent, respectively. The proximal marker of the stent is noted (arrow). D : Native angiogram after successful deployment of a Neuroform Atlas $(4.5 \times 30 \mathrm{~mm})$ stent from another distal branch to the proximal vessel via the aneurysm neck. The proximal marker of the Solitaire stent is noted (arrow). E : Another reconstructed flat panel detector CT image after deployment of both stents and microcatheter selection in the aneurysm dome showing adequate neck coverage without crossing of the stents. The proximal marker of the stent is noted (arrow). $F$ : Final native angiogram after coil packing in the aneurysm showing minimal neck remnant and herniated coil loops without flow disturbance. Proximal and distal markers of Solitaire AB (white arrows) and Neuroform Atlas (black arrows) stents are indicated.

plications during and after the procedures during 5 months follow-up with dual anti-platelet medications.

\section{DISCUSSION}

According to a meta-analysis of 27 studies on 750 aneurysms treatment using the Y-stent technique by Cagnazzo et $\mathrm{al}^{2}{ }^{2}$, the crossing Y-stent technique provides better aneurysmal neck coverage and branch preservation and shows enhanced long-term durability of aneurysm occlusion after coil packing. For the crossing Y-stent technique, a closed-cell type
Enterprise stent (45\%) has been most widely used, followed by the open-cell type Neuroform stent $(31.3 \%)^{2}$. Most cases were performed using two same-type stents (closed/closed-cell and open/open-cell stents, $87.5 \%$ ) while hybrid techniques using open-cell and closed-cell stent combinations were performed in $12.5 \%$.

However, the difficulty of passing the second microcatheter through the interstices of the first stent and possible deformity or dislocation of the first stent can be a significant burden, especially for beginners. The inevitable intersection point of the two stents commonly causes sandglass-like deformity or instent stenosis and poor vessel wall apposition of the second 
stent, resulting in reduced aneurysmal neck coverage. The tangled stent struts in the central vessel lumen hinder microcatheter selection inside the aneurysm sac and prohibit endothelialization, resulting in life-long elevated risk of acute or delayed thrombosis ${ }^{6,7)}$. These structural characteristics explain the thromboembolic complications (6.5\%), delayed complications after 30 days (2.1\%), and chronic in-stent stenosis $(2.3 \%)^{2}$. To reduce these disadvantages, nonoverlapping $\mathrm{Y}$ stenting or T-stenting techniques have been reported; crossing or kissing Y-stent techniques using lower profile stents, such as the LVIS Jr. (MicroVention-Terumo, Tustin, CA, USA) or Acclino (Acandis, Pforzheim, Germany), have been published recently ${ }^{1,3,8)}$. However, overall treatment-related complications (8.9\%) prove the continued existence of technical difficulties and risks of acute or delayed thromboembolic complications with these techniques ${ }^{2}$. Recently developed devices with distinct structures, such as the PulseRider (Cerenovus, Irvine, CA, USA) and pCONus (phenox GmbH, Bochum, Germany), are still unfamiliar to most neurointerventionists ${ }^{9,11)}$.

The Solitaire AB stent with full retrievability and an approximately $1-\mathrm{cm}$ long proximal tapered end has been used less frequently in Y-stenting than other stents for coil embolization assistance ${ }^{5)}$. We believe that these special features could enable us to avoid crossing of the stents and reduce the overlapping surface area. By appropriate installment of the Solitaire stent after meticulous measurement using 3D reconstruction software, we can expect to deploy the second stent easily, without crossing. As a result, there will be no in-stent stenosis or neck coverage loss in the second stent, which can contribute to easier microcatheter engagement to the aneurysm dome and reduce the risk of vessel injury and acute or delayed thrombosis. Owing to its full retrievability, we can repeat and re-adjust the installment until it is properly placed after confirmation of its deployment status using rotational angiogram or vasoCT.

We can jail the microcatheter and partially deploy the frame coil to reduce the difficulties and risks of microcatheter selection after the original crossing Y stenting. Partial frame coil deployment before stent deployment can even demonstrate the aneurysmal neck protection effects of stents during stent deployment (Fig. 1D). However, microcatheter jailing requires sufficient parent vessel diameter, which is often not achieved at the distal cerebral arteries. An important merit of this technique is that fewer difficulties can be encountered during mi- crocatheter selection into the aneurysmal sac because there is no crossing of stent struts, particularly in the center of the neck (Fig. 1C). The ease of microcatheter selection even after deployment of two stents is helpful, especially when the diameter of the parent vessel lumen is not sufficient for catheter jailing during the stent deployment step.

This technique also has some limitations. When the aneurysmal neck is very wide or the angles of the two branches are acute, there can be some stent-void parts in the aneurysmal neck, especially in the central area of the neck. We believe, however, that even in such a situation, there will be no great difficulty during coil framing and filling because this stenting technique can reduce the dome/neck ratio of aneurysms. Other assistance techniques such as the multiple catheter technique can also be helpful in such cases. We can also assume that the tapered proximal end portion of the deployed Solitaire stent might directly and precisely engage the origin of the other branch, and thus, we would be unable to avoid crossing of the second stent (Fig. 1E). In this structure, we can expect the proximal tapered end of the Solitaire stent to be aligned with the aneurysmal neck surface. Thus, it can induce better neck coverage, and we might expect to frame and fill coils without second stent deployment in such cases. This is somewhat similar to the upper part of the T-PulseRider installment ${ }^{9)}$. If coil framing and filling were not possible with the Solitaire stent deployment state, we would retrieve the Solitaire stent and try to deploy the stent differently by turning the stent system 180 degrees before insertion. It is theoretically evident that the possibility of the tapered proximal end directly engaging the opposite branch origin is not high.

\section{CONCLUSION}

This novel noncrossing Y-stent technique using the taper proximal end of the Solitaire AB stent can be considered an alternative to the original crossing Y-stent technique with fewer shortcomings, as well as similar neck coverage and branch preservation during coil embolization of broad neck bifurcation aneurysms. 


\section{CONFLICTS OF INTEREST}

No potential conflict of interest relevant to this article was reported.

\section{INFORMED CONSENT}

This type of study does not require informed consent.

\section{AUTHOR CONTRIBUTIONS}

\author{
Conceptualization : HJK \\ Data curation : HJK \\ Formal analysis : $\mathrm{HJK}$ \\ Funding acquisition : HJK, HSK \\ Methodology: HJK \\ Project administration : HJK, HSK \\ Visualization: HJK \\ Writing - original draft : HJK \\ Writing - review \& editing: JWL, HSB
}

\section{ORCID}

Hyon-Jo Kwon https://orcid.org/0000-0003-1077-2461

Jeong-Wook Lim https://orcid.org/0000-0003-1395-3145

Hyoung Soo Byoun https://orcid.org/0000-0003-2301-5988

Hyeon-Song Koh https://orcid.org/0000-0003-2659-5535

\section{- Acknowledgements}

This work was supported by research fund of Chungnam National University.

\section{References}

1. Brassel F, Melber K, Schlunz-Hendann M, Meila D : Kissing-Y stenting for endovascular treatment of complex wide necked bifurcation aneurysms using Acandis Acclino stents: results and literature review. J Neurointerv Surg $8:$ 386-395, 2016

2. Cagnazzo F, Limbucci N, Nappini S, Renieri L, Rosi A, Laiso A, et al. : Ystent-assisted coiling of wide-neck bifurcation intracranial aneurysms: a meta-analysis. AJNR Am J Neuroradiol 40 : 122-128, 2019

3. Cho YD, Park SW, Lee JY, Seo JH, Kang HS, Kim JE, et al. : Nonoverlapping Y-configuration stenting technique with dual closed-cell stents in wide-neck basilar tip aneurysms. Neurosurgery 70(2 Suppl Operative) : 244-249, 2012

4. Granja MF, Cortez GM, Aguilar-Salinas P, Agnoletto GJ, Imbarrato G, Jaume A, et al. : Stent-assisted coiling of cerebral aneurysms using the Y-stenting technique: a systematic review and meta-analysis. J Neurointerv Surg 11 : 683-689, 2019

5. Klisch J, Clajus C, Sychra V, Eger C, Strasilla C, Rosahl S, et al. : Coil embolization of anterior circulation aneurysms supported by the Solitaire AB Neurovascular Remodeling Device. Neuroradiology 52 : 349-359, 2010

6. Kono K, Shintani A, Yoshimura R, Okada H, Tanaka Y, Fujimoto T, et al. : Triple antiplatelet therapy with addition of cilostazol to aspirin and clopidogrel for Y-stent-assisted coil embolization of cerebral aneurysms. Acta Neurochir (Wien) 155 : 1549-1557, 2013

7. Kono $K$, Terada $T$ : Feasibility of insertion of a microcatheter through a $Y$ stent in coil embolization of cerebral aneurysms and its detailed geometry by micro-computed tomography. Acta Neurochir (Wien) 156 : 39-43, 2014

8. Samaniego EA, Mendez AA, Nguyen TN, Kalousek V, Guerrero WR, Dandapat $\mathrm{S}$, et al. : LVIS Jr device for Y-stent-assisted coil embolization of wide-neck intracranial aneurysms: a multicenter experience. Interv Neurol 7 : 271-283, 2018

9. Spiotta AM, Derdeyn CP, Tateshima S, Mocco J, Crowley RW, Liu KC, et al. : Results of the ANSWER trial using the PulseRider for the treatment of broad-necked, bifurcation aneurysms. Neurosurgery $81: 56-65$, 2017

10. Thorell WE, Chow MM, Woo HH, Masaryk TJ, Rasmussen PA : Y-configured dual intracranial stent-assisted coil embolization for the treatment of wide-necked basilar tip aneurysms. Neurosurgery 56 : 1035-1040; discussion 1035-1040, 2005

11. Ulfert C, Pfaff J, Schönenberger S, Bösel J, Herweh C, Pham M, et al. : The pCONus device in treatment of wide-necked aneurysms : technical and midterm clinical and angiographic results. Clin Neuroradiol 28 : 47-54, 2018

12. Xue G, Zuo Q, Duan G, Zhang X, Zhao R, Li Q, et al. : Dual stent-assisted coil embolization for intracranial wide-necked bifurcation aneurysms: a single-center experience and a systematic review and meta-analysis. World Neurosurg 126 : e295-e313, 2019 